\title{
Direct observation of the phase space footprint of a painting injection in the Rapid Cycling Synchrotron at the Japan Proton Accelerator Research Complex
}

\author{
P. K. Saha, ${ }^{*}$ Y. Shobuda, H. Hotchi, N. Hayashi, and T. Takayanagi \\ Japan Atomic Energy Agency (JAEA), Tokai, Ibaraki 319-1195, Japan \\ H. Harada \\ Hiroshima University, Higashi Hiroshima, Hiroshima 739-8526, Japan \\ Y. Irie \\ High Energy Accelerator Research Organization (KEK), Tsukuba, Ibaraki 305-0801, Japan
}

(Received 14 January 2009; published 30 April 2009)

\begin{abstract}
The 3 GeV Rapid Cycling Synchrotron (RCS) at Japan Proton Accelerator Research Complex is nearly at the operational stage with regard to the beam commissioning aspects. Recently, the design painting injection study has been commenced with the aim of high output beam power at the extraction. In order to observe the phase space footprint of the painting injection, a method was developed utilizing a beam position monitor (BPM) in the so-called single pass mode. The turn-by-turn phase space coordinates of the circulating beam directly measured using a pair of BPMs entirely positioned in drift space, and the calculated transfer matrices from the injection point to the pair of BPMs with several successive turns were used together in order to obtain the phase space footprint of the painting injection. There are two such pairs of BPMs placed in two different locations in the RCS, the results from which both agreed and were quite consistent with what was expected.
\end{abstract}

DOI: 10.1103/PhysRevSTAB.12.040403

PACS numbers: 29.20.dk, 29.27.Ac

\section{INTRODUCTION}

The beam commissioning of the $3 \mathrm{GeV}$ Rapid Cycling Synchrotron (RCS) at Japan Proton Accelerator Research Complex (J-PARC) started in October 2007, and is nearly in the transition to the user operation stage [1]. As designed, the RCS is currently acting as an injector for the $50 \mathrm{GeV}$ main ring (MR) as well as a stable beam source for the Material and Life science Facility (MLF), although with only the low beam power and duty required by the successive facilities in their initial beam commissioning. The design goal with the RCS is to achieve $1 \mathrm{MW}$ of output beam power with injection and extraction energies of 0.4 and $3 \mathrm{GeV}$, respectively, having $8.3 \times 10^{13}$ protons per pulse at a repetition rate of $25 \mathrm{~Hz}$ [2]. However, the injection energy at present is $181 \mathrm{MeV}$, but the output beam power with the $3 \mathrm{GeV}$ extraction is expected to be around $0.6 \mathrm{MW}$.

In order to realize such a high power beam at the extraction, the RCS was designed to control the beam density at the lower energies so as to mitigate the space charge force by utilizing the phase space painting injection in the transverse direction and longitudinal painting with $\mathrm{rf}$ operation in the longitudinal direction. A uniform spatially distributed beam in the transverse plane with a higher

\footnotetext{
*Corresponding author. saha.pranab@j-parc.jp
}

bunching factor (ratio of average current to the peak current) can then be obtained utilizing painting injection schemes in the transverse and the longitudinal planes, respectively $[3,4]$. In addition, the number of average foil hits by the circulating beam due to the multiturn injections can be significantly reduced utilizing a transverse painting injection, which results in a large fraction of the uncontrolled beam loss caused by the nuclear scattering together with the large angle multiple Coulomb scattering to eventually be reduced [5]. A uniform beam distribution utilizing painting injection is preferable in keeping the beam density as small as possible in order to avoid damaging the neutron production mercury target with such a high power beam of $1 \mathrm{MW}[6,7]$. This then increases the usefulness of painting injection from various points of interest.

Precise practical knowledge of the painting injection mechanism is therefore necessary as it can provide direct feedback to the effort of aiming the design goal. The basic principle of painting injection in the transverse direction is to control the beam distribution through phase space painting. In simulation, sophisticated phase space control patterns are used so as to meet the requirements. Thorough knowledge of phase space information with painting injection in real time could therefore lead to direct control of the phase space. Unfortunately, however, this type of study is still basically limited to simulations or at best some simple comparisons of the beam profiles obtained in simulations and those measured with beams utilizing painting injec- 
tions $[8,9]$. The present work describes how phase space control can be realized on-line and provide direct feedback to the painting injection study.

The present work was a challenge for such a real time application of the phase space control, and hence an attempt in the RCS was made to directly observe the phase space footprint of the painting injection in the transverse direction. This was carried out utilizing the turn-by-turn phase space information of the central trajectory of the circulating beam measured with a pair of BPMs located in drift space and calculating the transfer matrices from the injection point to the pair of BPMs together with a few successive turns. There were two such pairs of BPMs in the RCS ring. The first pair was located in the extraction straight section, while the second pair was located in the rf straight section. A single pass mode of BPMs had already been utilized for a variety of purposes that include measuring the injection orbit, correcting the injection error, beam dynamics studies, etc. [10-15], but were yet to have been applied in directly measuring the phase space footprint of the painting injection in a proton machine. On the other hand, the fact that both BPMs of each pair were positioned in drift space was a strong advantage of the present attempt. The phase space coordinates of the central trajectory of the circulating beam could be directly measured without use of any models, assumptions, or extrapolation. The phase space coordinates of the central trajectory of the circulating beam were the first ingredient for the present issue. Directly measuring the phase space footprint of the painting injection in the present work is an advanced approach to using single pass mode BPMs, and achieved very successful and accurate results. The present method can play an important role in efficient elaborate painting injection studies in the RCS in achieving the design goal of $1 \mathrm{MW}$.

The layout of this paper is as follows. A general overview of the RCS including the injection system and painting injection process in given in Sec. II, a method of analyzing the BPM single pass data in obtaining the turnby-turn beam position is briefly described in Sec. III, the present method is given in Sec. IV, the results of the reconstructed footprint of the painting injection presented in Sec. V, error analysis is described in Sec. VI, a discussion is provided in Sec. VII, and finally a summary is given in Sec. VIII.

\section{PAINTING INJECTION IN RCS}

Figure 1 shows the general layout of the RCS. The RCS is a threefold symmetric lattice with a circumference of 348.333 meters [2]. Each superperiod consists of two 3DOFO (defocusing and focusing periodic cell structure) arc modules with missing bends and 3-DOFO insertions. The three insertions are named I, E, and R and are dispersion free. The injection and transverse collimation systems are located in the I insertion, the extraction system in the $\mathrm{E}$

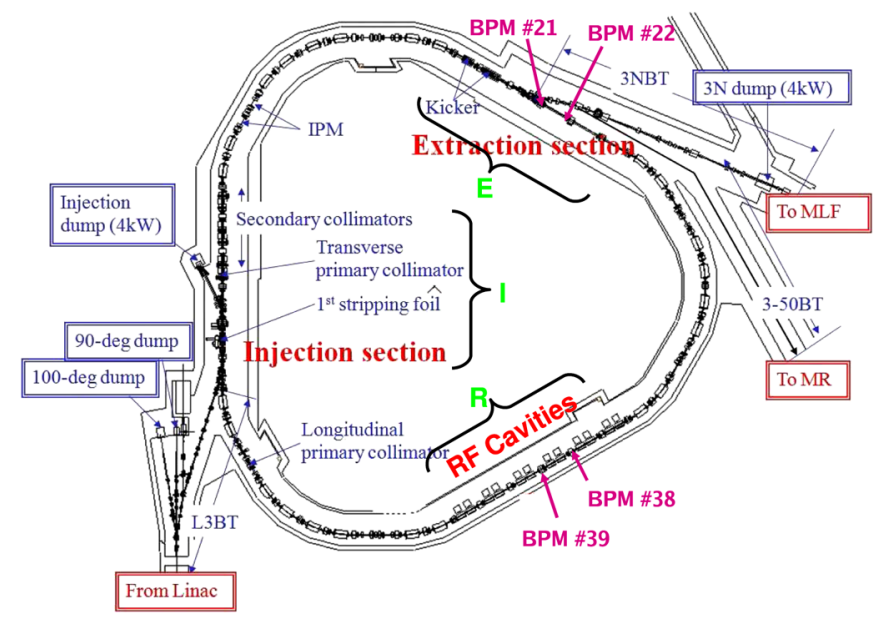

FIG. 1. (Color) Layout of RCS. Three insertions are named as I, $\mathrm{E}$, and $\mathrm{R}$. The injection and transverse primary collimator systems are located in the I insertion, the extraction system in the $\mathrm{E}$ insertion, and the $\mathrm{rf}$ cavities are in the $\mathrm{R}$ insertion. Two pairs of BPMs positioned completely in drift space were used in the single pass mode in the present painting injection study. The first pair is located in the extraction straight section (BPM \#21 and \#22), while the second pair is located in the rf straight section (BPM \#38 and \#39).

insertion, and the rf cavities in the $\mathrm{R}$ insertions. The $\mathrm{H}^{-}$ charge-exchange injection system occupies about onethird of the I insertion, while the collimation system occupies the rest of the I insertion.

Figure 2 shows the general layout of the $\mathrm{H}^{-}$chargeexchange injection system of RCS. The latest specifications of all the magnets in the injection system are summarized in Refs. [16,17]. There are a total of eight closed bump magnets in the horizontal direction, with four of them (SB1 4) being known as shift bump magnets. They are positioned in the uninterrupted drift space between two quadrupole magnets (QFL and QDL) and have the role of forming a constant closed bump orbit during injection. The other four (PBH1 4) are known as horizontal paint bump magnets, with the first two being located upstream of the QFL and the other two downstream of the QDL. Two vertical paint bump magnets named PBV1 and PBV2 are placed in the L3BT (linac-to-3 GeV-beamtransport) line for painting in the vertical direction. A couple of BPMs (K-BPM, I-BPM) and several multiwire profile monitors (MWPMs) [18] are placed almost between the magnets as a beam diagnostics system.

Figure 3 shows a schematic view of the painting injection process in the horizontal (up) and vertical (down) directions. The numerical parameters are for a typical painting area of $216 \pi \mathrm{mm}$ mrad. Painting injection in the horizontal plane is performed by sweeping the closedorbit utilizing the decay wave form of the magnetic field, while painting in the vertical plane is done by directly sweeping the injection beam angle utilizing the decay wave form patterns of the two vertical paint bump magnets 


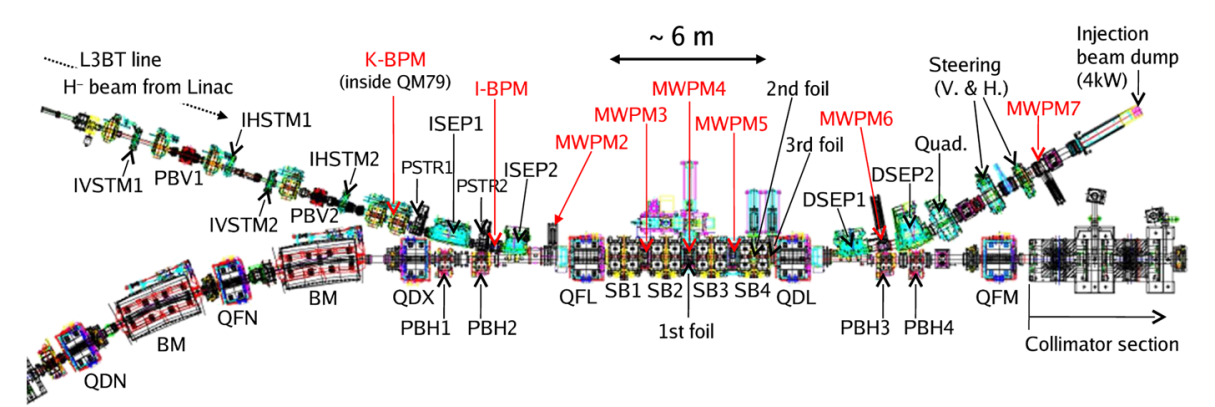

FIG. 2. (Color) Layout of RCS injection and successive H0 dump line. Four horizontal shift bump magnets (SB1 $\sim 4)$ produce a constant closed orbit, while in addition, another four paint bump magnets (PBH1 $\sim 4)$ in the horizontal direction and two vertical paint bump magnets $(\mathrm{PBV} 1 \sim 2)$ in the vertical direction produce time dependent fields for the painting injection. The beam diagnostics system consists of a couple of BPMs (K-BPM, I-BPM) and several MWPMs (multiwire profile monitors) placed almost between the magnets.
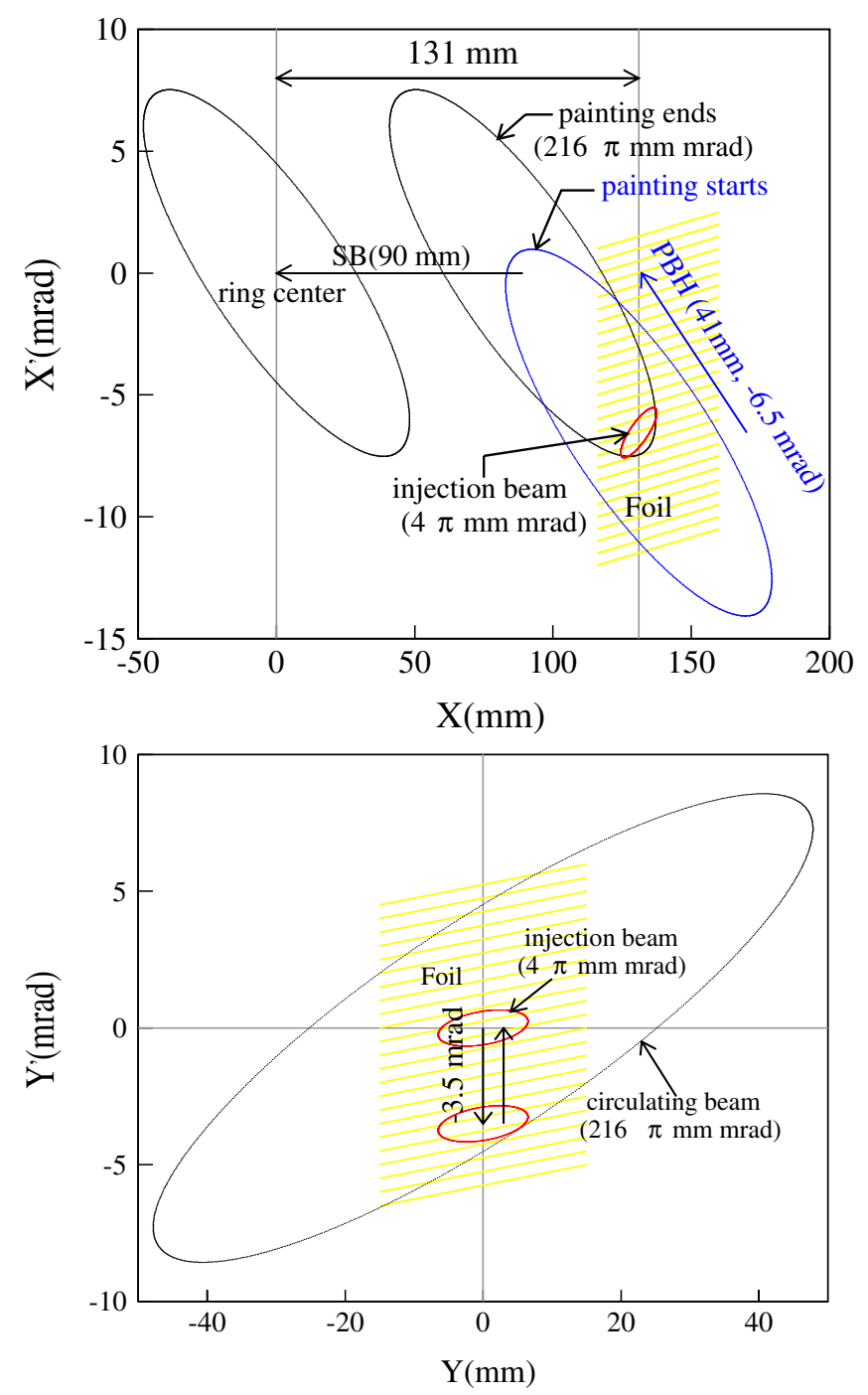

FIG. 3. (Color) Schematic diagrams of the painting injection process performed in the RCS in the horizontal (top) and vertical (bottom) directions with typical design parameters. In the vertical direction, the injection beam can be painted either center to outside or outside to center for so-called correlated or anticorrelated painting, respectively.
(PBV1 2). The first (PBV1) is a main painting magnet located at almost a phase difference of $180^{\circ}$ from the charge-exchange foil, while the second (PBV2) is an auxiliary type used to adjust the phase difference of the main painting magnet to the foil position. The painting injection in the horizontal direction was basically designed for center to outside, while in the vertical direction the injection beam can be painted either center to outside or outside to center by changing the excitation patterns of the vertical painting magnets in so-called correlated or anticorrelated painting, respectively, as can be surmised using Fig. 3. The decay waveform pattern of the paint bump magnets is basically a square root function of time [3]. In the beginning, the painting injection was performed using a correlated painting process, in which trajectories of the injection beam at the injection point with respect to the circulating orbit as a function of time during the painting injection period become

$$
\begin{gathered}
\mathbf{X}_{\text {paint }}=\mathbf{X}_{\max }\left(\sqrt{\frac{t_{1}}{T_{1}}}\right), \\
\mathbf{X}_{\text {paint }}^{\prime}=-\mathbf{X}_{\max }^{\prime}\left(\sqrt{\frac{t_{1}}{T_{1}}}\right), \\
\mathbf{Y}_{\text {paint }}=0, \\
\mathbf{Y}_{\text {paint }}^{\prime}=-\mathbf{Y}_{\max }^{\prime}\left(\sqrt{\frac{t_{1}}{T_{1}}}\right),
\end{gathered}
$$

where $\mathbf{X}_{\max }, \mathbf{X}_{\max }^{\prime}$, and $\mathbf{Y}_{\max }^{\prime}$ determine the top excitation level of the paint bump of the desired painting area. $T_{1}$ corresponds to a paint injection time of $500 \mu \mathrm{s}$, and $t_{1}$ is the time step starting from 0 to the maximum of $T_{1}$.

The design painting injection area with the MLF beam is $216 \pi \mathrm{mm}$ mrad and $144 \pi \mathrm{mm}$ mrad with the MR beam. Because of the narrower physical aperture of the MR beam transport line compared to that of the MLF beam transport line, the painting injection for the MR beam is limited to 
the smaller area of $144 \pi \mathrm{mm}$ mrad in the RCS. For that reason, two pulse steering magnets (PSTR1,2) are placed in the RCS injection line and made available in the next phase together with a linac beam energy of $400 \mathrm{MeV}$. A change in the painting area from one cycle to another in the horizontal direction could then be performed by changing the inclination of the injection beam. The excitation levels of the shift bump magnets are simultaneously changed in order to keep the unchanged injection beam position on the foil. The painting area in the vertical direction is changeable using the two vertical paint bump magnets (PBV1,2).

\section{ANALYSIS OF THE BEAM POSITION MONITOR DATA}

Before describing the principles of the present method, a brief description will be provided here concerning the analysis of the BPM data in obtaining turn-by-turn beam position information in the single pass mode. The usual method of obtaining COD (closed-orbit distortion) will also be briefly explained.

There are a total of 54 BPMs in the RCS that are used for various measurements including the COD [19]. Two pairs of them were completely within the drift space (no elements in between), as can be seen in Fig. 1. The first pair (BPM \#21 and \#22), located in the extraction straight section, were named "pair 1," while the second pair (BPM \#38 and \#39) located in the rf straight section were named "pair 2." The two BPMs in each pair were about $5.5 \mathrm{~m}$ apart. For the present purpose of studying the painting injection, these two pairs of BPMs were used in the so-called single pass mode to obtain turn-by-turn beam position information of the central trajectory of the circulating beam. The turn-by-turn beam position is one of the key information points in the present method and thus the utmost efforts were made to extract the correct information.

The sensor head of a BPM is of the electrostatic induction type and each BPM has four electrodes (a, b, c, and d) at approximately $45^{\circ}$ angles. The COD is measured at $1 \mathrm{~ms}$ intervals during the total cycle of $20 \mathrm{~ms}$. The data for the first $100 \mu \mathrm{s}$ (about 50 turns around the injection period) of each interval is used for this purpose, corresponding to $4 \mathrm{k}$ sample points. Fast Fourier transform analysis is then used to determine the amplitudes of the induced voltages for four channels, denoted here as $V_{a}, V_{b}, V_{c}$, and $V_{d}$, by detecting the peak value within a certain range. The horizontal $(x)$ and vertical $(y)$ positions are then calculated by using the following equations:

$$
\begin{gathered}
u=a_{1} \frac{V_{a}-V_{b}}{V_{a}+V_{b}}+b_{1}, \quad v=a_{2} \frac{V_{c}-V_{d}}{V_{c}+V_{d}}+b_{2}, \\
{\left[\begin{array}{l}
u \\
v
\end{array}\right]=\left[\begin{array}{cc}
\cos \theta & -\sin \theta \\
\sin \theta & \cos \theta
\end{array}\right]\left[\begin{array}{l}
x \\
y
\end{array}\right],}
\end{gathered}
$$

where $a_{1}, b_{1}, a_{2}$, and $b_{2}$ are the BPM parameters deter- mined using the calibrations and measurements of the laser tracker after installation, and $\theta$ is the rotation angle of the BPM electrodes $\left(\sim 45^{\circ}\right)$.

However, in the single pass mode $V_{a}, V_{b}, V_{c}$, and $V_{d}$ are needed to precisely determine turn by turn. As an example the analysis procedure for one electrode will be described here.

Figure 4 shows the observed signal as a function of time for electrode "a" with the data being for the first several turns. As seen in the figure, the signal contains a certain level of noise, although quite low, with the baseline being shifted due to the electrostatic nature of the BPM. A schematic drawing of the pulse function with notations is provided in the upper part of the figure and used in the following analytic derivations.

First, the single pulse given in the range of $t_{\text {ini }}$ to $t_{\text {fin }}$ is considered (refer to Fig. 4). If the single pulse function $f(t)$ is assumed to be periodic along the time axis with periodicity $T$, it can be then expanded using the Fourier coefficients of $c_{n}$ and $d_{n}$ as

$$
f(t)=\sum_{n=0}^{\infty} c_{n} \cos \frac{2 n \pi}{T} t+\sum_{n=1}^{\infty} d_{n} \sin \frac{2 n \pi}{T} t .
$$

Averaging $f(t)$ over $t$ from initial $t_{\text {ini }}$ to the final time $t_{\text {fin }}$ enables the $n=0$ th order coefficient to be obtained. By extracting the $n=0$ th order term from Eq. (7), the base line shift from the signal can be subtracted. Furthermore, the assumption is made that the $n=1$ st order term domi-

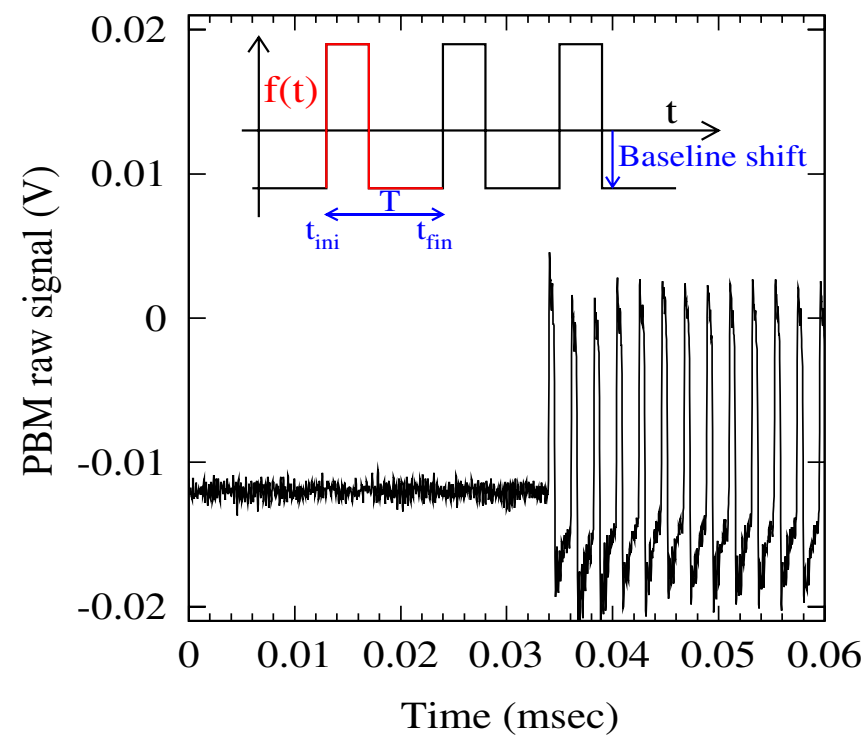

FIG. 4. (Color) Time domain signal from one of the four electrodes of BPM \#21. The data is for the first several turns of the injection energy. The RCS was in beam storage mode with beam energy of $181 \mathrm{MeV}$, where only a single pulse with peak current of about $25 \mathrm{~mA}\left(\sim 1 \times 10^{11}\right.$ particles $)$ from the linac was injected into RCS. The schematic drawing of the pulse function $f(t)$ with some notations given in the upper part was used in the derivations presented in this section. 
nates when compared to the higher order terms. The signal without its noise $\tilde{f}(t)$ can be approximated as

$$
\tilde{f}(t) \simeq c_{1} \cos \frac{2 \pi}{T} t+d_{1} \sin \frac{2 \pi}{T} t \equiv V_{a} \sin \left(\frac{2 \pi t}{T}+\varphi\right),
$$

for which the voltage amplitude $V_{a}$ for the pulse can be determined.

Since the sampling frequency of a BPM is $40 \mathrm{MHz}$, the above-mentioned $\tilde{f}(t)$ gets digitized at the $25 \mathrm{~ns}$ step. When the number of points in pulse length $T$ is $N$, they can be summarized using

$$
\left[\begin{array}{c}
\tilde{f}\left(t_{1}\right) \\
\vdots \\
\tilde{f}\left(t_{N}\right)
\end{array}\right]=\left[\begin{array}{c}
V_{a} \sin \left(\frac{2 \pi}{T} t_{1}+\varphi\right) \\
\vdots \\
V_{a} \sin \left(\frac{2 \pi}{T} t_{N}+\varphi\right)
\end{array}\right]=\left[\begin{array}{cc}
\sin \left(\frac{2 \pi}{T} t_{1}\right) & \cos \left(\frac{2 \pi}{T} t_{1}\right) \\
\vdots & \vdots \\
\sin \left(\frac{2 \pi}{T} t_{N}\right) & \cos \left(\frac{2 \pi}{T} t_{N}\right)
\end{array}\right]\left[\begin{array}{c}
V_{a} \cos (\varphi) \\
V_{a} \sin (\varphi)
\end{array}\right]
$$

Application of the least square method to the data results in

$$
\begin{aligned}
\cos (\varphi) & =\frac{\sum_{i=1}^{N} \cos ^{2}\left(\omega t_{i}\right) \sum_{j=1}^{N} \sin \left(\omega t_{j}\right) \tilde{f}\left(t_{j}\right)-\sum_{i=1}^{N} \sin \left(\omega t_{i}\right) \cos \left(\omega t_{i}\right) \sum_{j=1}^{N} \cos \left(\omega t_{j}\right) \tilde{f}\left(t_{j}\right)}{V_{a}\left\{\sum_{i=1}^{N} \sin ^{2}\left(\omega t_{i}\right) \sum_{j=1}^{N} \cos ^{2}\left(\omega t_{j}\right)-\left[\sum_{i=1}^{N} \sin \left(\omega t_{i}\right) \cos \left(\omega t_{i}\right)\right]^{2}\right\}} \\
\sin (\varphi) & =\frac{-\sum_{i=1}^{N} \sin \left(\omega t_{i}\right) \cos \left(\omega t_{i}\right) \sum_{j=1}^{N} \sin \left(\omega t_{j}\right) \tilde{f}\left(t_{j}\right)+\sum_{i=1}^{N} \sin ^{2}\left(\omega t_{i}\right) \sum_{j=1}^{N} \cos \left(\omega t_{j}\right) \tilde{f}\left(t_{j}\right)}{V_{a}\left\{\sum_{i=1}^{N} \sin ^{2}\left(\omega t_{i}\right) \sum_{j=1}^{N} \cos ^{2}\left(\omega t_{j}\right)-\left[\sum_{i=1}^{N} \sin \left(\omega t_{i}\right) \cos \left(\omega t_{i}\right)\right]^{2}\right\}}
\end{aligned}
$$

where $\omega=2 \pi / T$. By dividing Eq. (11) by (10) the phase $\varphi$ is calculated using

$$
\varphi=\tan ^{-1}\left[\frac{-\sum_{i=1}^{N} \sin \left(\omega t_{i}\right) \cos \left(\omega t_{i}\right) \sum_{j=1}^{N} \sin \left(\omega t_{j}\right) \tilde{f}\left(t_{j}\right)+\sum_{i=1}^{N} \sin ^{2}\left(\omega t_{i}\right) \sum_{j=1}^{N} \cos \left(\omega t_{j}\right) \tilde{f}\left(t_{j}\right)}{\sum_{i=1}^{N} \cos ^{2}\left(\omega t_{i}\right) \sum_{j=1}^{N} \sin \left(\omega t_{j}\right) \tilde{f}\left(t_{j}\right)-\sum_{i=1}^{N} \sin \left(\omega t_{i}\right) \cos \left(\omega t_{i}\right) \sum_{j=1}^{N} \cos \left(\omega t_{j}\right) \tilde{f}\left(t_{j}\right)}\right],
$$

Substituting Eq. (12) into Eq. (9) results in amplitude $V_{a}$ of electrode "a" being obtained. Similarly, with the same pulse, amplitudes $V_{b}, V_{c}$, and $V_{d}$ for the other three electrodes are also obtained. The beam positions were then calculated using Eqs. (5) and (6). By repeating this procedure, the beam positions for the other successive pulses can then be obtained.

\section{PRESENT METHOD}

Once the central trajectory of the circulating beam has been obtained turn by turn by utilizing the procedures explained in the previous section, the present method is rather simple and straightforward. The phase space footprint of the injection beam at the injection point can then be obtained using the following equation:

$$
\left[\begin{array}{l}
X_{\mathrm{BPM}} \\
X_{\mathrm{BPM}}^{\prime}
\end{array}\right]=\left[\mathbf{M}^{n}\right]\left[\mathbf{M}_{1 \text { st foil } \rightarrow \mathrm{BPM}}\right]\left[\begin{array}{c}
X_{\text {foil }} \\
X_{\text {foil }}^{\prime}
\end{array}\right],
$$

where $X_{\mathrm{BPM}}$ and $X_{\mathrm{BPM}}^{\prime}$ are the phase space coordinates of the central trajectory of the circulating beam measured turn by turn using BPM pair 1 or 2 . Once the beam positions have been ascertained by the two BPMs, the phase space coordinates of the central trajectory of the circulating beam are calculated at the first BPM of each pair. The measured COD at each BPM is subtracted and the geometrical length from one BPM to the other of the pair is used to calculate the phase space coordinates of the circulating beam. $X_{\text {foil }}$ and $X_{\text {foil }}^{\prime}$ are defined as the initial phase space coordinates of the injection beam at the injection point and must then be obtained. $\mathbf{M}_{1 \text { st foil } \rightarrow \text { BPM }}$ is the transfer matrix from the injection point to the corresponding BPM pair. $\mathbf{M}^{n}$ is a transfer matrix for $n$ turns of the ring starting from the 1 st BPM of each pair, which can be expressed using the following equation:

$$
\mathbf{M}^{n}=\left[\begin{array}{cc}
\cos (\phi)+\alpha \sin (\phi) & \beta \sin (\phi) \\
-\gamma \sin (\phi) & \cos (\phi)-\alpha \sin (\phi)
\end{array}\right],
$$

where $\phi=2 \pi n \Delta \mu, n$ corresponds to the turn number, and $\Delta \mu$ denotes the fraction of the betatron tune. $\alpha, \beta$, and $\gamma\left(=\frac{1+\alpha^{2}}{\beta}\right)$ are Twiss parameters. In a similar manner, replacing $Y$ to $X$ in Eq. (13) and calculating the transfer matrices for the vertical direction results in the phase space footprint in the vertical direction being obtained. Experimental data for up to the first ten turns are used in the analysis.

In order to calculate the transfer matrices, a realistic model using strategic accelerator design (SAD) [20] was constructed by introducing all possible error sources, and was exactly the same as used in the beam commissioning. The measured optics was first reproduced by the calculated one with slightly changing the strength of the quadrupole 
magnets as reported in more detail in Ref. [1]. As expected, all three straight sections were found to be dispersion free and thus there was no effect of the dispersion on the present method as both pairs of the BPMs are positioned in straight sections. This therefore made measuring the optics an important issue in the present method. However, using a realistic model enables the difference to be made narrower, as proved true in the present case, where the reconstructed $X_{\text {foil }}$ and $X_{\text {foil }}^{\prime}$ in Eq. (13) were found to only vary by $\pm 0.7 \mathrm{~mm}$ and $\pm 0.06 \mathrm{mrad}$, respectively, when using quadrupole parameters without correction. Worthy of mention here is that the edge focusing effect of the bump magnets especially, the shift bump magnets, with which the vertical transfer matrix is subject to change, was taken into consideration in calculating the vertical transfer matrix.

As for the experimental condition, the RCS was used in beam storage mode with a beam energy of $181 \mathrm{MeV}$, where only a single pulse from the linac was injected into the RCS in order to clearly observe the betatron oscillations. The peak current of the linac beam was therefore set as high as $25 \mathrm{~mA}\left(\sim 1 \times 10^{11}\right.$ particles $)$ in order to gain the signal over noise (refer to Fig. 4).

Before use in the painting injection study, the present method was first applied in two basic purposes in order to verify the validity and accuracy, which will be discussed in the following subsections.

\section{A. Correction of injection error}

The first application was in correcting the injection error, which in another sense was in principle verification of the present method. The injection error means here the offset of the injection beam with respect to the closed orbit at the injection point, and thus that correction means matching the injection beam to the closed orbit in both the horizontal and vertical directions in decreasing the size of the betatron oscillations. Equation (13) clearly identified the injection errors, which were very accurately corrected with a single trial using injection septa (ISEP1,2). The betatron oscillations were reduced to a minimum, which was confirmed by looking at mountain views of the beam profiles measured by the ionization profile monitor (IPM) [21] both before and after the correction. The beam profiles both before and after the injection error correction can be referred to in Ref. [1]. The results obtained from both pairs were found to be very consistent with each other and it is worth mentioning here that, before establishment of the present method, the injection error used to be gradually corrected by examining mountain views of the beam profiles measured by IPM as a function of the injection parameters and separately in the horizontal and vertical directions. This was very time consuming taking about 6 hours for the full correction, while the present method only takes a few minutes as practically only a single shot beam is sufficient to determine the injection error and for correction then using ISEP1,2.

\section{B. Calibration of the paint bump magnets top excitation levels}

The second application was in calibrating the top excitation levels of all the paint bump magnets for a desired painting area, which in fact was the basis and first part of the painting injection study. Once the top excitation level is determined, the decay pattern for a painting injection time of $500 \mu \mathrm{s}$ can then be easily made using design functions, which are basically the square root function of time as discussed in Sec. II. In the horizontal direction, the calibration was carried out by exciting the paint bump magnets with a constant at the top for the desired painting injection area, which can be considered the equivalent of an offset injection. Figure 5 shows a demonstration of the horizontal paint bump patterns used in that calibration with a constant at the top (red curve). The shift bump magnets were also excited together with constant at the top (black curve). The decay pattern of the paint magnets is given with the blue curve.

Examining the reconstructed phase space coordinates at the injection point obtained using Eq. (13) lead to the paint bump magnets being calibrated and thus the desired phase space coordinates obtained, which was then confirmed by directly measuring the injection orbit with MWPM3 and

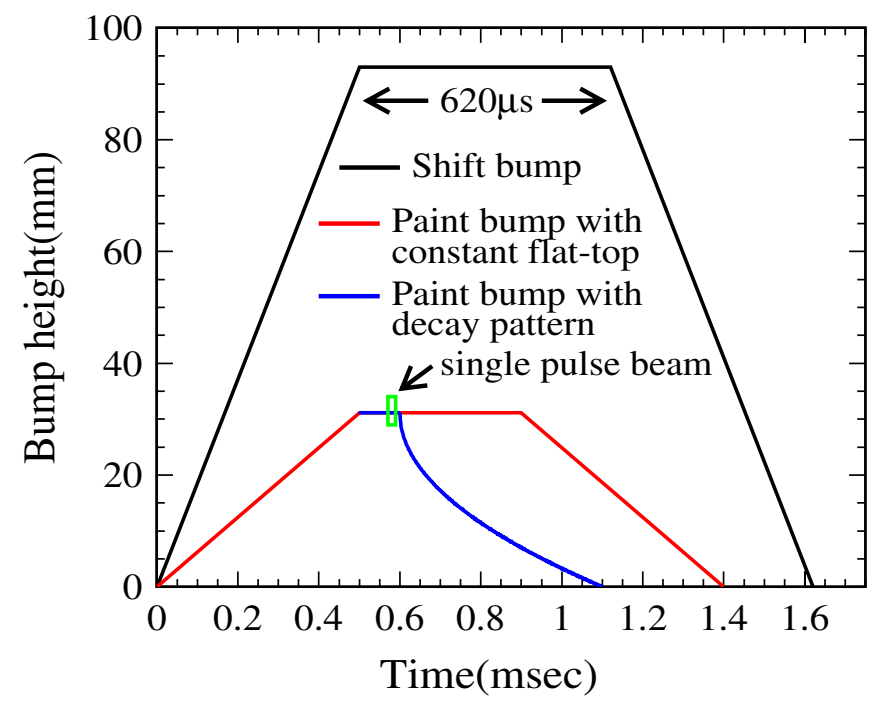

FIG. 5. (Color) Schematic view of the bump patterns used for different injection modes. The shift bump magnets had constant at the top, as given by the black curve, used for the center injection. The paint bump magnets were excited at the top of the shift bump magnets with a constant at the top (red curve) or decay pattern (blue curve) in accordance with the present painting injection study. The sum of the height of the shift bump and paint bump magnets was $124 \mathrm{~mm}$ for a painting area of $100 \pi \mathrm{mm} \mathrm{mrad}$. The footprint of the painting injection was studied at six different timings by delaying (or shifting) the decay pattern of the paint bump magnets (blue curve) at steps of $100 \mu \mathrm{s}$. A single pulse beam from the linac was injected into the RCS. The timing of the beam with respect to the shift bump was unchanged, as revealed in the figure. 
MWPM4. In the horizontal direction, the relative difference of the beam positions measured for the painting and center injection using MWPM3 and MWPM4 provided the relative inclination of the injection beam, where the beam position at the injection point could be calculated using the position measured by MWPM4 and the inclination, and thus together providing the phase space coordinates of the injection beam at the injection point (1st foil). The injection point is located about $56 \mathrm{~mm}$ downstream of the MWPM4. It is important to note here that the injection beam inclination at the injection point for the center injection orbit was adjusted to be almost zero (matching the closed orbit). In the vertical direction, calibration took place mainly by measuring the vertical beam position of the injection beam directly with MWPM3 and MWPM4, and this was because the vertical paint bump magnets are positioned in the injection line. However, the present method was also used to confirm it.

The numerical results of the calibration are summarized in Table I. As revealed by the table, the results obtained from both pairs also agreed well with each other, and moreover, were consistent with the direct measurements obtained using MWPM. In addition, the normalized phase space plots shown in Fig. 6 were made using turn-by-turn data measured by BPM pair 1, and thus the emittance formed by the central trajectory of the circulating beam was calculated. Using the calculated Twiss parameters of the circulating beam and considering the measured emittance $(3.6 \pi \mathrm{mm} \mathrm{mrad}$ in $3 \sigma)$ and Twiss parameters of the

TABLE I. Numerical results of the reconstructed phase space coordinates at the injection point for a painting area of $100 \pi \mathrm{mm}$ mrad in the horizontal and vertical directions after calibration of the top excitation levels of the paint bump magnets. The paint bump magnets were excited with constant at the top for the desired painting area given as by "Target," where the phase space coordinates at the injection point had been reconstructed utilizing Eq. (13). The results obtained with both pairs were in good agreement and were also basically consistent with direct measurements made using the MWPMs. After the paint bump top excitation level had been efficiently calibrated, the decay wave forms of the magnetic field as a function of time were then easily made.

\begin{tabular}{lcc}
\hline \hline $\begin{array}{l}\text { Paint area } \\
(\pi \mathrm{mm} \text { mrad })\end{array}$ & $\begin{array}{c}\text { Injection }\left(\mathrm{X}, \mathrm{X}^{\prime}\right) \\
(\mathrm{mm}, \mathrm{mrad})\end{array}$ & Comment \\
\hline $\begin{array}{l}\text { Horizontal } \\
100\end{array}$ & $(31.1,-4.4)$ & Target \\
& $(30.6,-4.69)$ & Eq. (13) with pair 1 \\
& $(30.1,-4.52)$ & Eq. (13) with pair 2 \\
& $(31.2,-4.61)$ & With MWPM \\
Vertical & $\left(\mathbf{Y}, \mathbf{Y}^{\prime}\right)$ & Target \\
100 & $(0,-1.91)$ & Eq. (13) with pair 1 \\
& $(-1.0,-2.01)$ & Eq. (13) with pair 2 \\
& $(-0.8,-2.1)$ & With MWPM \\
\hline \hline
\end{tabular}

injection beam, the emittance formed by the central trajectory of the circulating beam for a full painting area of $100 \pi \mathrm{mm} \mathrm{mrad}$ was expected to be $75 \pi \mathrm{mm} \mathrm{mrad}$ in
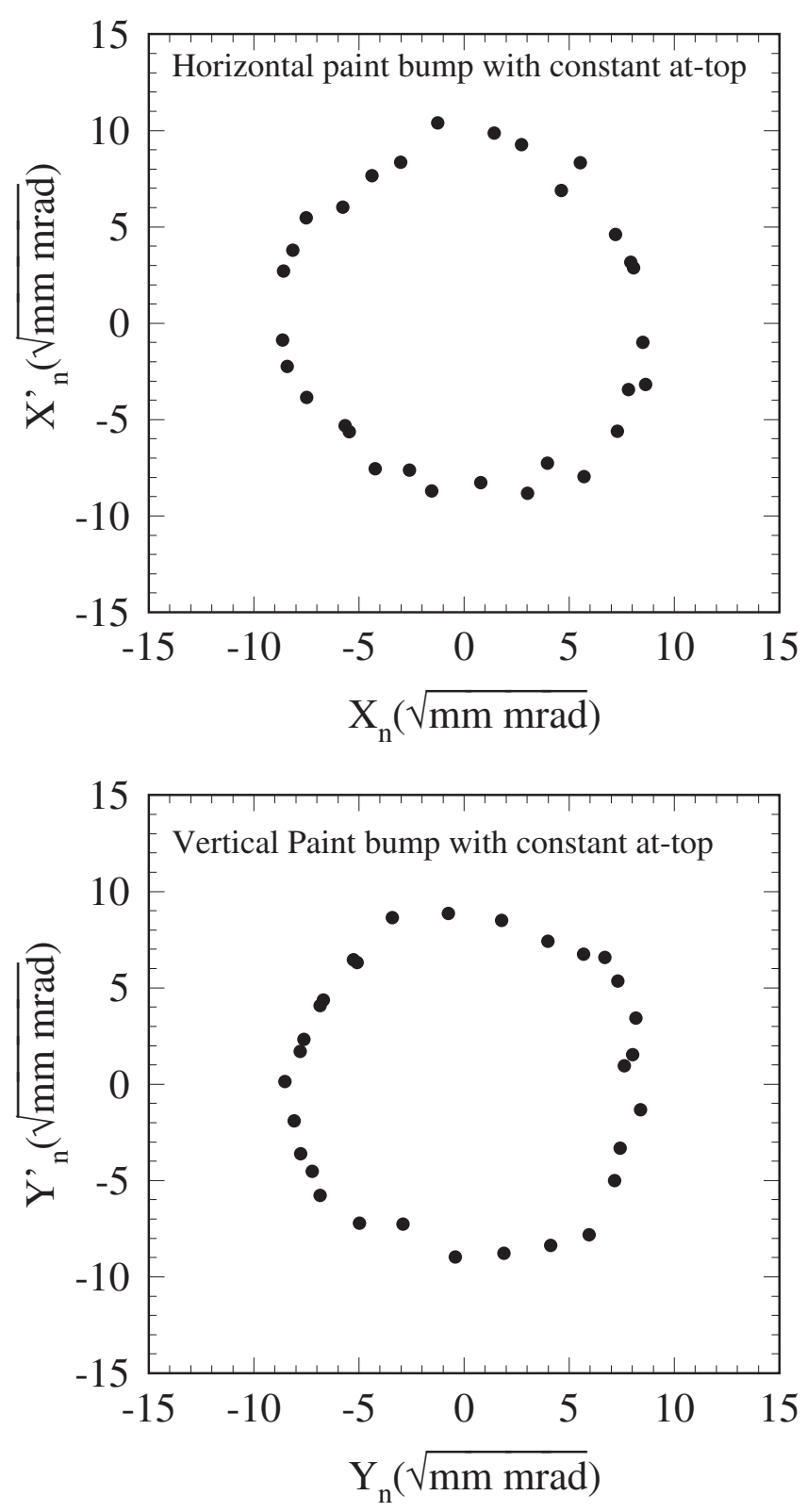

FIG. 6. Normalized phase space plots of the central trajectory of the circulating beam obtained from the turn-by-turn beam positions measured by a pair of BPMs over the first 30 turns. Measurement took place separately in the horizontal and vertical directions, as given in the upper and lower figures, respectively. The paint bump magnets were excited with constant at the top for a full painting area of $100 \pi \mathrm{mm}$ mrad. The emittance formed by the central trajectory of the circulating beam was expected to be $75 \pi \mathrm{mm}$ mrad. As expected, each of the phase space plots was circular, and the emittances formed by the central trajectories of the circulating beam were then calculated from the area of the circles and found to be 78.8 \pm 2.1 and $74.8 \pm$ $2.1 \pi \mathrm{mm} \mathrm{mrad}$ in the horizontal and vertical directions, respectively. 
both horizontal and vertical directions. From statistical analysis of the normalized phase space, those were found to be $78.8 \pm 2.1 \pi \mathrm{mm} \mathrm{mrad}$ and $74.1 \pm 2.1 \pi \mathrm{mm} \mathrm{mrad}$ in the horizontal and vertical directions, respectively, and thus providing quite consistent with what was expected.

It was also easy to understand here too that, without use of the present method, such calibration would have been rather difficult, particularly in the horizontal direction. Both $\mathrm{X}$ and $\mathrm{X}^{\prime}$ are parameters with definite values for a painting injection, where the injection and the circulating orbit should match at the start of the painting. If there is any mismatch, calibration of paint bump top excitation level for the desired painting is in practice very hard to do by examining the beam profile measured with an IPM.

The decay wave form patterns of the paint bump magnetic field were then made for use in a correlated painting study. The trajectories at the injection point with respect to time were expected to change, as revealed by Eqs. (1)-(4). As a first step, the study was comparatively performed using the smaller painting area of $100 \pi \mathrm{mm}$ mrad, with which $\mathbf{X}_{\max }, \mathbf{X}_{\max }^{\prime}$, and $\mathbf{Y}_{\max }^{\prime}$ in Eqs. (1), (2), and (4) were $31.1 \mathrm{~mm}, 4.4 \mathrm{mrad}$, and $1.91 \mathrm{mrad}$, respectively, as can also be seen in Table I.

\section{FOOTPRINT OF THE PAINTING INJECTION}

In order to observe the phase space footprint of the painting injection during $500 \mu \mathrm{s}$, data was obtained at six different times $(\mathrm{t} 0 \sim \mathrm{t} 5)$ by delaying (or shifting) the decay patterns (the blue curve in Fig. 5 for the horizontal direction, but similar for the vertical direction) at steps of $100 \mu \mathrm{s}$. The time stamp t0 corresponded to the start of the painting (center of the circulating beam phase space), while 55 was that at the end of painting (outside the circulating beam phase space). Here also a single pulse from the linac through the painting injection orbit was injected into the RCS. The study was first done separately in the horizontal and vertical directions and then together in the horizontal and vertical directions for a correlated painting injection provided by Eqs. (1)-(4).

Figure 7 shows the reconstructed phase space footprint of the painting injection done separately in the horizontal (top) and vertical directions (bottom) for a painting area of $100 \pi \mathrm{mm} \mathrm{mrad}$, with the same legends being applicable in both figures. The phase space of the circulating beam at the injection point is given by the large ellipse depicted with black line for the desired painting area, where the red ellipse is the measured injection beam, which was found to be $3.6 \pi \mathrm{mm} \operatorname{mrad}(3 \sigma)$. The results obtained using BPM pair 1 are given as blue dots, while the green dots depict those obtained using the data of BPM pair 2. The data point depicted by the open square at 55 was obtained as a direct MWPM measurement using the same principle explained earlier. The expected footprints of the painting injection obtained using Eqs. (1)-(4) are given as red dots, and thus the footprints observed were expected to follow the linear red lines reproduced in all intermediate states. As revealed by the above figure, the present method enabled the phase space footprints during the painting injection to be accurately observed, although in the vertical direction the reconstructed footprints for some intermediate time stamps
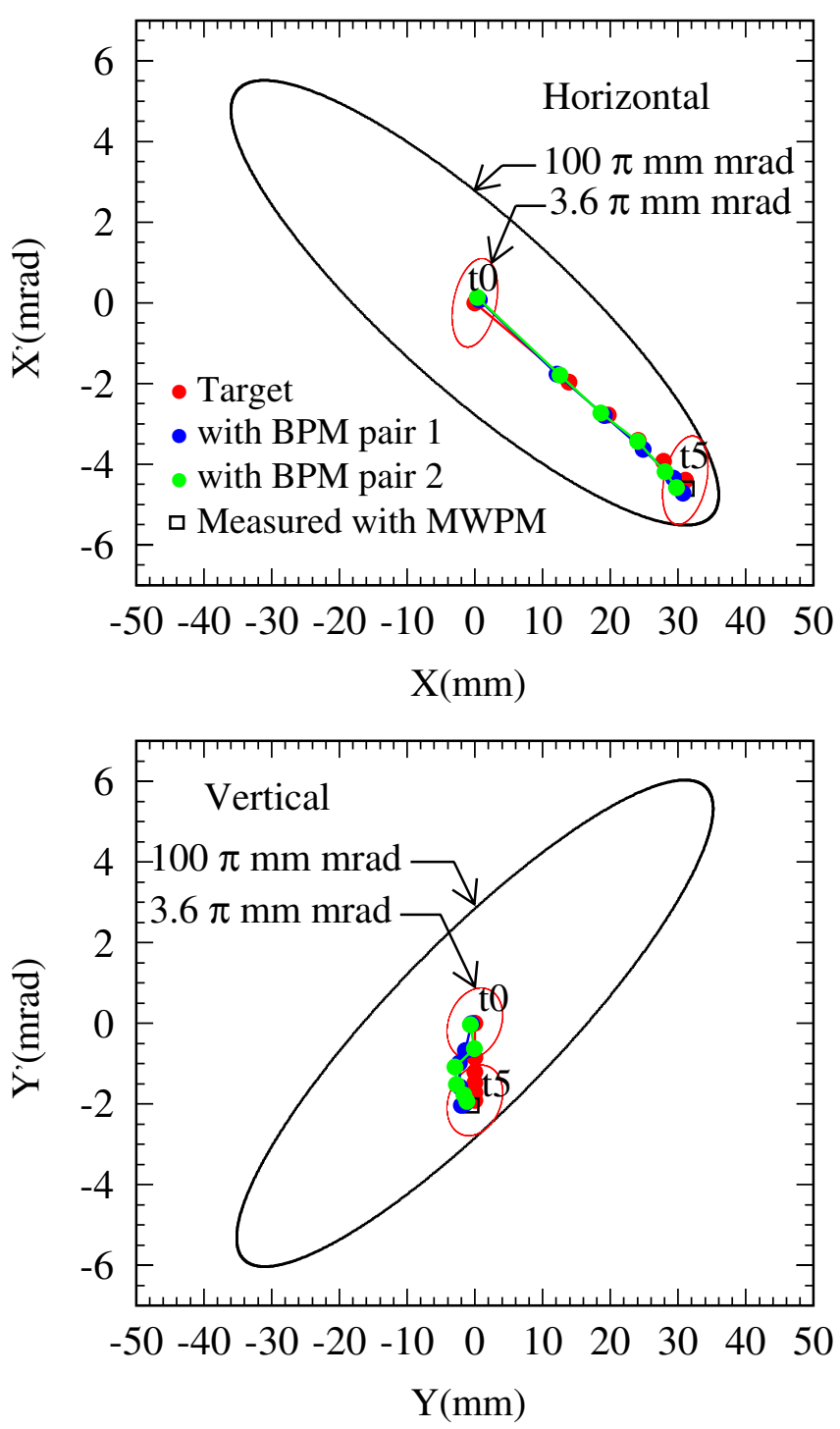

FIG. 7. (Color) Reconstructed phase space footprint obtained using Eq. (13) for a painting area of $100 \pi \mathrm{mm} \mathrm{mrad}$ done separately in the horizontal (top) and vertical directions (bottom). Measurements took place at six different timings of the paint bump decay pattern covering the entire period of $500 \mu \mathrm{s}$ by delaying (or shifting) the bump patterns in steps of $100 \mu \mathrm{s}$. Time stamp t0 corresponds to the start of painting, while t5 denotes the end. The legend is the same for both figures. The results from both pairs were consistent with each other and were found to nicely fit as expected on the linear red lines by reproducing all the intermediate states, although with slight deviations with some intermediate time stamps in the vertical direction. The error bars in the horizontal direction are included within the size of the symbols, as discussed in the latter part of Sec. VI (Fig. 9). 
were a bit zigzag, but did finally reach the expected goal at t5. The reason for that may have been due to fluctuations in the vertical paint bump pattern, in particular with the 2nd vertical paint bump (PBV2), which was operated at rather low current when compared to its maximum capability. The 2nd vertical paint bump magnet was used as an auxiliary in order to adjust the phase difference from the 1st painting magnet to the injection point. Fine-tuning of the
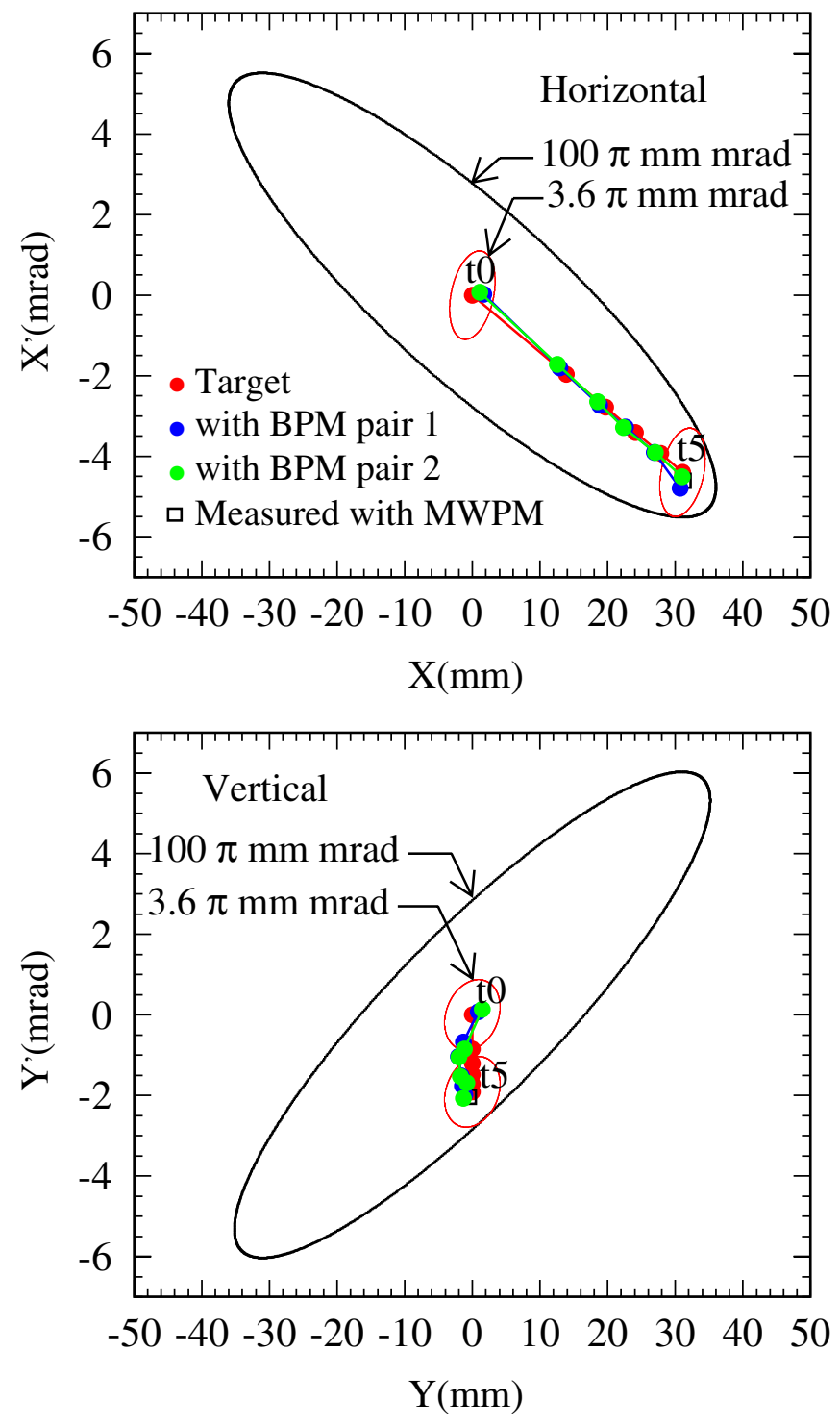

FIG. 8. (Color) Reconstructed phase space footprint obtained using Eq. (13) for a correlated (center to outside for both the horizontal and vertical direction) painting injection of $100 \pi \mathrm{mm}$ mrad. Here also the measurement was done with six different timings of the paint bump decay pattern of $500 \mu \mathrm{s}$. The legend is the same for both figures. The results obtained here were also found very consistent and followed the expected linear red line in each direction. The results obtained here can be compared with those obtained for the same painting area of $100 \pi \mathrm{mm}$ mrad but done separately in the horizontal and vertical directions, given in Fig. 7, and found quite consistent. bump patterns would possibly improve that fluctuation and thus the necessary steps are under consideration. Estimating the injection error due to the fluctuations of the paint bump magnets together with the shift bump magnets is presented in Sec. VI.

The reconstructed phase space footprint obtained for a correlated painting injection of $100 \pi \mathrm{mm}$ mrad is shown in Fig. 8 for the horizontal (top) and vertical (bottom) directions. Here also, by ignoring the fluctuations in the vertical intermediate states, the obtained results proved very consistent with what was expected to be very similar to the results obtained separately in the horizontal and vertical directions as shown in Fig. 7. In addition to the accuracy of the present method, it also reflected the system performance in terms of stability. Direct observation of the phase space footprint of the painting injection helped to justify the decay wave form patterns of the paint bump magnets, and was thus used in the real painting injection study in RCS [1].

\section{ERROR ESTIMATION}

As the present method utilizes turn-by-turn information of the beam position measured using pairs of BPMs, the quality of the results obtained, given above, depends on several factors including resolution of the BPMs. Other considerable factors include the fluctuation of the shift bump flattop and fluctuation of the paint bump patterns, along with the stability of the injection beam. Although not in very great detail but qualitative analysis was done to estimate all these individual factors as presented in this section. From the turn-by-turn beam position measured by the BPMs, the resolution of all four BPMs used in the present method was found to be about $1.2 \mathrm{~mm}(\mathrm{rms})$ with the present experimental conditions (one single pulse injection). However, multiturn injection was discovered to be much better $(0.25 \mathrm{~mm})$, with the beam intensity due to accumulation being about 5 times higher when compared to the single pulse injection used in the painting injection study. The fluctuation of the shift bump flattop was found to be within $\pm 0.3 \%$, for which the injection error was estimated to be $\pm 0.6 \mathrm{~mm}$. The fluctuation of the horizontal paint bump magnets was found be about $\pm 0.5 \%$ at the top of the excitation level, but was slightly worse at the lower excitation level. The injection error with the fluctuation at the top excitation level was estimated to be about $\pm 1.2 \mathrm{~mm}$ and $\pm 0.11 \mathrm{mrad}$ in the worst combination of all four magnets.

However, fluctuations with the vertical paint bump magnets were much worse, with the second one (VPB2), in particular. By taking into account the fluctuations of the vertical paint bump magnets, offline analysis revealed that fluctuation of the injection beam position and inclination at the injection point in the vertical direction was a maximum of $\pm 1.2 \mathrm{~mm}$ and $\pm 0.18 \mathrm{mrad}$, respectively, relatively large when compared to the horizontal direction. 
Stability with the injection beam was quite good in terms of energy as well as beam position, and thus the effect was considered to be negligibly small. The energy and the beam position jitters monitored near the RCS injection were found to be about $20 \mathrm{keV}$ and $60 \mu \mathrm{m}$, respectively [22]. The deviations of the observed footprints from the expected ones as seen in Figs. 7 and 8, can be explained within the fluctuations of all shift bump and paint bump magnets, including the resolution of BPMs as presented above.

That said however, an attempt was made to directly extract the effect of the overall fluctuation in the horizontal direction from the data by repeating the measurement for a painting area of $100 \pi \mathrm{mm}$ mrad with a constant at the top (refer to Table I). 111 measurements were made, and phase space coordinates $\left(\mathrm{X}\right.$ and $\left.\mathrm{X}^{\prime}\right)$ at the injection point through use of Eq. (13) extracted for each measurement. The results obtained were plotted in a histogram, which is shown in

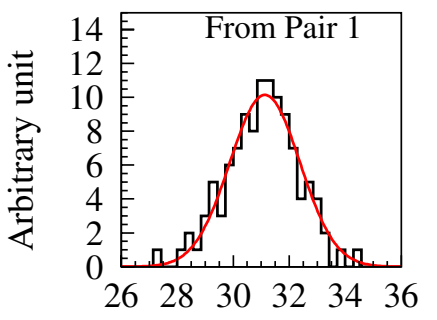

$\mathrm{X} \_$injection $(\mathrm{mm})$
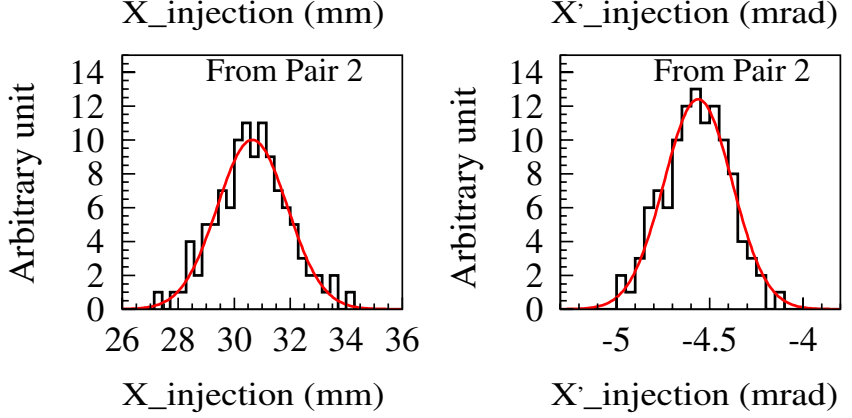

FIG. 9. (Color) The overall error of the result obtained using the present method was estimated by repeating measurements (paint bump with constant at the top for a painting area of $100 \pi \mathrm{mm}$ mrad, refer to Table I) 111 times. The phase space coordinates at the injection point were obtained using Eq. (13) for each measurement and plotted as histograms. The upper two figures represent the results obtained using data from pair 1, whereas the lower two figures are for those obtained using data from pair 2 . The mean value and the width were obtained by fitting each distribution to a Gaussian function (solid red lines). The mean value of $X$ and $X^{\prime}$ from pair 1 were then found to be $31.1 \mathrm{~mm}$ and $-4.66 \mathrm{mrad}$, respectively, while those from pair 2 were found to be $30.7 \mathrm{~mm}$ and $-4.52 \mathrm{mrad}$, respectively. The rms widths from each pair were exactly the same at $1.2 \mathrm{~mm}$ and $0.19 \mathrm{mrad}$ for $\mathrm{c}$, respectively. The mean values of the results from both pairs were thus found consistent using the single measurement presented in Table I, where the overall error in rms for the reconstruction of the painting injection footprint can be considered to be $\pm 1.2 \mathrm{~mm}$ and $\pm 0.19 \mathrm{mrad}$ for $\mathrm{X}$ and $\mathrm{X}^{\prime}$, respectively.
Fig. 9. The top two figures give the reconstructed phase space coordinates at the injection point obtained using the data from pair 1 , while the bottom two figures are those obtained using the data from pair 2 . The mean value and width in each case were obtained by fitting each distribution to a Gaussian function. The mean of $X$ and $X^{\prime}$ from pair 1 was found to be $31.1 \mathrm{~mm}$ and $-4.66 \mathrm{mrad}$, respectively, with deviations in rms of $1.2 \mathrm{~mm}$ and $0.19 \mathrm{mrad}$, respectively. Here also the results from pair $2(30.7 \mathrm{~mm}$ and $-4.56 \mathrm{mrad}$ ) were very similar to those from pair 1 , as revealed in the figure. The mean values of $\mathrm{X}$ and $\mathrm{X}^{\prime}$ obtained here were compared to a single measurement presented in Table I, done in the same experimental conditions and for the same painting area of $100 \pi \mathrm{mm}$ mrad in the horizontal direction (pair 1: $30.6 \mathrm{~mm}$ and $-4.69 \mathrm{mrad}$; pair 2: $30.1 \mathrm{~mm}$ and $-4.52 \mathrm{mrad}$ ). The similarity of the results obtained from both pairs, and consistency with what was expected as well as with the single measurement, again here reflected the accuracy of the present method. In order to draw a conclusion concerning the overall error in reconstruction of the phase space footprint using the present method, the deviations of $\mathrm{X}$ and $\mathrm{X}^{\prime}$ found using the 111 measurements ( $\pm 1.2 \mathrm{~mm}$ and $\pm 0.19 \mathrm{mrad}$ ) could be used, and were included within the size of each symbol of the observed footprint in the horizontal direction.

\section{DISCUSSION}

The present method used calculated transfer matrices from the injection point to the corresponding BPM pair with few successive turns as given by Eqs. (13) and (14). For that purpose, a realistic model based on the SAD simulation tool was constructed, which included all possible error sources. The model successfully reproduced the measured optics of the ring. Successful correct observations of the phase space footprint, in addition to the present method, also proved the accuracy of the calculated transfer matrices.

The observed phase space footprints were quite consistent with expectation, although slight fluctuations were observed in the vertical direction. As mentioned earlier, the fluctuations in the vertical direction were mainly related to the fluctuations of the second vertical paint bump magnets. Controlling the decay wave form patterns of those two magnets could be used to improve that situation, and thus the necessary steps are to be taken. The argument could be made that the transfer matrix during the painting injection, in the vertical direction, in particular (when done in both planes together), changes as a function of time due to the edge effect of the decaying horizontal paint bump magnets. However, compared to the shift bump magnets the effect of the paint bump magnets should be insignificant. This is because they are located comparatively wider apart and operated at a much lower strength when compared to the shift bump magnets, as revealed in Figs. 2 and 5 , respectively. In practice this can also be seen by compar- 
ing the bottom of Fig. 7 to the bottom of Fig. 8. In the former case, painting injection only in the vertical direction was performed and thus the horizontal paint bump magnets were kept off but the reconstructed footprints were found to be almost identical to that with the latter case. Analysis also proved the effect to be negligible, and the calculated transfer matrices with and without the edge effect of the paint bump magnets were found to only slightly change. The result was that reconstructed phase space footprints in the vertical direction were found to only change by $0.2 \mathrm{~mm}$ and $0.04 \mathrm{mrad}$.

The merit of the present method was not only limited to direct phase space control of painting injection, but can also be considered an efficient tool in correcting injection errors as well as calibrating the top excitation level of the paint bump magnets. Any change in the injection beam or the ring parameter requires the injection error to be corrected, which connects to the next step of the RCS beam commissioning. In addition, calibrating the paint bump top excitation level is needed for the desired painting injection with any new parameter of the injection or circulating beam. For those purposes, information on the circulating beam just after the injection and in addition turn by turn is very important. The turn-by-turn phase space information measured directly by utilizing a pair of BPMs in single pass mode is thus one of the main advantages of the present method. Another merit is that it is very fast, and thus efficiently used in every run cycle for basic purposes that include the painting injection study. The results obtained from both pairs were very similar to each other and quite consistent with what was expected, thus reflecting the accuracy of the present method.

\section{SUMMARY}

The phase space footprint of a painting injection in the transverse plane was directly observed using BPMs in single pass mode. The phase space information of the central trajectory of the circulating beam measured turnby-turn with a pair of BPMs and calculated transfer matrices were used together to obtain the phase space coordinates at the injection point. The transfer matrices were calculated using the SAD simulation tool. The present method was proved a powerful and timely tool for correcting injection errors in the RCS. Only a few minutes, rather than the few hours it used to take before establishment of the present method, were needed, and it is efficiently using every run cycle. Accurate calibration of the top excitation level of the paint bump magnets was also very efficient, which is the basic start of a painting injection but apparently rather hard to do efficiently as well as time consuming before the present method was made available.

The phase space footprints observed in the present study were found to be very consistent with what was expected as well as direct measurements made using MWPM. Precise understanding of the painting process in real time in this way could also lead to direct control of the transverse phase space and feedback towards achieving higher beam power through a painting injection.

The present work is a first and successful application of BPM in single pass mode with the painting injection study, and is thus a new and significant advance in actual painting injection study. Such an effective and efficient feedback for use in realistic painting injection studies could not be realized through conventional methods such as measuring the beam profile with an IPM or other beam profile monitor. Direct realization of the phase space control in reality needs to be achieved in the first stage of a painting injection study; hence, the belief that the present result will encourage utilization of the application with other machines where $\mathrm{H}^{-}$painting injection is performed as well as consideration to the proposal of any new $\mathrm{H}^{-}$charge-exchange injection scheme.

\section{ACKNOWLEDGMENTS}

The authors would like to acknowledge the tremendous efforts of all the members of the J-PARC project in their progress with the beam commissioning that enabled painting injection in the RCS to start at such an early stage. The efforts of the J-PARC control group, particularly Mr. H. Takahashi in dealing with the huge amount of BPM single pass data, are highly appreciated. The authors are also grateful to Professor N. Yamamoto, Professor T. Toyama, Professor K. Oide, Mr. F. Noda, and Mr. T. Nakamura from IHI for their large amount of support which included valuable discussions concerning the analysis of the BPM single pass data. Finally, the authors would also like to thank Dr. M. Kinsho for his continuous support and encouragement.

[1] H. Hotchi, in Proceedings of the 42nd ICFA Advanced Beam Dynamics Workshop (HB2008), Nashville, Tennessee, 2008, OPL02.

[2] JAERI Technical Report No. 2003-044 and KEK Report No. 2002-13.

[3] Yukihide Kamiya, in Proceedings of the Particle Accelerator Conference, Chicago, USA, 1989 (IEEE, New York, 1989), pp. 660-662.

[4] Fumihiko Tamura, Masanobu Yamamoto, Masahito Yoshii, Chihiro Ohmori, Masahiro Nomura, Alexander Schnase, Makoto Toda, Hiromitsu Suzuki, Taihei Shimada, Keigo Hara, and Katsushi Hasegawa (to be published).

[5] P. K. Saha, in Proceedings of the 42nd ICFA Advanced Beam Dynamics Workshop (HB2008), Nashville, Tennessee, 2008 (Ref. [1]), WGC05.

[6] Shin-ichiro Meigo, Fumiaki Noda, Syuichi Ishikura, Masatoshi Futakawa, Shinichi Sakamoto, and Yujiro Ikeda, Nucl. Instrum. Methods Phys. Res., Sect. A 562, 569 (2006). 
[7] Hiroyuki Kogawa, Syuichi Ishikura, Hiroshi Sato, Masahide Harada, Shunichi Takatama, Masatoshi Futakawa, Katsuhiro Haga, Ryutaro Hino, Shinichiro Meigo, Fujio Maekawa, and Yujiro Ikeda, J. Nucl. Mater. 343, 178 (2005).

[8] S. M. Cousineau, Nucl. Instrum. Methods Phys. Res., Sect. A 561, 297 (2006).

[9] B. Jones, D. J. Adams, and C. M. Warsop, in Proceedings of the 11th European Particle Accelerator Conference, Genoa, 2008 (EPS-AG, Genoa, Italy, 2008), pp. 35873589.

[10] C. Gardner and L. Ahrens, IEEE Trans. Nucl. Sci. 32, 1888 (1985).

[11] T. Honda, M. Katou, A. Ueda, M. Tadano, Y. Kobayashi, and T. Mitsuhashi, in Proceedings of the Particle Accelerator Conference, Vancouver, BC, Canada, 1997 (IEEE, New York, 1997), pp. 2084-2086.

[12] P. Castro, J. Borer, A. Burns, G. Morpurgo, and R. Schmidt, in Proceedings of the Particle Accelerator Conference, Washington, DC, 1993 (IEEE, New York, 1993), pp. 2103-2105.

[13] M. Shirakata, H. Sato, T. Toyama, and K. Marutsuka, KEK Report No. 93-11, 1993.

[14] Y. Kobayashi, T. Mitsuhashi, A. Ueda, and T. Kasuga, Proceedings of the 5th European Particle Accelerator Conference, Sitges, Spain, 1996, pp. 1666-1668.

[15] A. Drago, M. N. Biagini, S. Guiducci, C. Milardi, M. Preger, C. Vaccarezza, and M. Zobov, in Proceedings of the 21st Particle Accelerator Conference, Knoxville, 2005 (IEEE, Piscataway, NJ, 2005), pp. 4326-4328.

[16] M. Yoshimoto, J. Kamiya, T. Takayanagi, M. Watanabe, O. Takeda, M. Kinsho, and Y. Irie, in Proceedings of the 10th European Particle Accelerator Conference, Edinburgh, Scotland, 2006 (EPS-AG, Edinburgh, Scotland, 2006), pp. 1768-1770.

[17] T. Takayanagi, J. Kamiya, M. Watanabe, Y. Yamazaki, Y. Irie, J. Kishiro, I. Sakai, and T. Kawakubo, IEEE Trans. Appl. Supercond. 16, 1358 (2006).

[18] S. Hiroki, N. Hayashi, M. Kawase, F. Noda, P. K. Saha, H. Sako, H. Takahashi, A. Ueno, Y. Arakida, S. Lee, and T. Toyama, in Proceedings of the 11th European Particle Accelerator Conference, Genoa, 2008 (Ref. [9]), pp. 1131-1133.

[19] N. Hayashi, S. Hiroki, R. Saeki, R. Toyokawa, K. Yamamoto, D. Arakawa, S. Hiramatsu, S. Lee, K. Satou, M. Tejima, and T. Toyama, in Proceedings of the 11th European Particle Accelerator Conference, Genoa, 2008 (Ref. [9]), pp. 1128-1130.

[20] See http://acc-physics.kek.jp/SAD/sad.html.

[21] K. Satou, N. Hayashi, S. Lee, and T. Toyama, in Proceedings of the 10th European Particle Accelerator Conference, Edinburgh, Scotland, 2006 (Ref. [16]), pp. 1163-1165.

[22] M. Ikegami, in Proceedings of the 42nd ICFA Advanced Beam Dynamics Workshop (HB2008), Nashville, Tennessee, 2008 (Ref. [1]), WGD02. 